\title{
Effect of Various Shapes of Rough Transverse Slider Bearing Equipped with Ferro-Lubricant on the Load-Tolerating Capability by Mathematical Modeling
}

\author{
G. C. Panchal ${ }^{1 *}$, H. C. Patel ${ }^{2}$, H. A. Patel ${ }^{3}$ \\ ${ }^{1}$ Department of Mathematics, Vishvakarma Government Engineering College, Chandkheda, \\ Zip-382424, Gujarat State, India \\ ${ }^{2}$ Indian Institute of Teacher's education, Gandhinagar, Zip-382016, Gujarat State, India \\ ${ }^{3}$ Department of Mathematics, Gujarat Power Engineering, and Research Institute, Mehsana, \\ Zip-382710, Gujarat State, India
}

Received 2 January 2021, accepted in final revised form 30 May 2021

\begin{abstract}
This paper investigates the effect of various film shapes of rough transverse slider bearing with Ferro-lubricant on the bearing system's load-tolerating capability (LTC). The current article describes the efforts to improve bearing's LTC by using a Ferro-lubricant as a nonNewtonian fluid and choosing the bearing's (piston ring's) proper geometrical shape in the I. C. Engine. The mathematical model and the numerical and graphical results reveal the facts about enhancing the bearing system's performance. Moreover, the adverse effect of roughness can be lessened to a certain range by growing the magnetic field's strength.
\end{abstract}

Keywords: Averaged Reynolds' type Eq. (ARTE); Slider bearing; Transverse roughness; Ferro-lubricant.

(C) 2021 JSR Publications. ISSN: 2070-0237 (Print); 2070-0245 (Online). All rights reserved. doi: http://dx.doi.org/10.3329/jsr.v13i3.51072 J. Sci. Res. 13 (3), 745-761 (2021)

\section{Introduction}

The classical lubrication theory establishes the prediction of the bearing's behavior in the sense of friction, wear, roughness pattern parameters (RPP), and load-tolerating capability for the case of Newtonian fluids. In the bearing's system, the roughness and lubricants play a crucial role in getting better performance.

Much research work is done to reduce friction and wear and enrich the performance in an internal combustion engine by design and mechanical point of view. Many investigators [1-3] analyzed the bearing's lifetime and performance influenced by friction, wear, lubricants, and surface roughness. The bearing's performance depends on different types of roughness and lubricant [4-7]. Many results showing the relationship among the essential parameters like bearing's LTC, mean, SD, skewness, and magnetization parameter are established.

\footnotetext{
*Corresponding author: gcpanchal@vgecg.ac.in
} 
The different types of material combinations can be a suitable option for the ferrofluid-based journal bearing system and enhance the lifetime of the bearing [8]. Some appropriate situations being generated due to the positive effect of ferrofluid help reimburse the low impact of roughness [9].

In these studies, the role of Christensen and Tonder [10-13] could not be undervalued who have formed ARTE by smearing the stochastic averaging procedure on" Reynolds' type Equation," which provides the mean-pressure more desirable than a local-pressure.

In the present article, the comparison between Plane Shape Slider Bearing (PSB), Hyperbolic Shape Slider Bearing (HSB), Exponential Shape Slider Bearing (ESB), and Secant Shape Slider Bearing (SSB) is analyzed. We used Simpson's 1/3-rule to evaluate the integrals while solving the modified ARTE. It is investigated how the RPP makes variations in the performance of the bearing system in various shapes. It gives the graphical comparison between altered bearing geometries or the geometric shapes (plane, exponential, hyperbolic, and secant) of piston-ring. Moreover, the adverse effect of roughness can be lessened to a specific range by growing the magnetic field's strength.

\section{Analysis}

The averaged Reynolds' type equation (1) that governs the mean pressure in a rough slider bearing is,

$$
\frac{\partial}{\partial x}\left[\varphi_{x} \frac{b_{T}^{3}}{12 \eta} \frac{\partial \bar{p}}{\partial x}\right]+\frac{\partial}{\partial y}\left[\varphi_{y} \frac{b_{T}^{3}}{12 \eta} \frac{\partial \bar{p}}{\partial y}\right]=\frac{V}{2} \frac{\partial \bar{b}_{T}}{\partial x}+\frac{V \sigma}{2} \frac{\partial \varphi_{S}}{\partial x}
$$

which was established by Patir [14] for two-dimensional flow in a slider bearing. Here $b$ is a local film thickness, $\overline{b_{T}}$ is average film thickness, $\eta$ is the viscosity of the lubricant, $\overline{\mathrm{p}}$ is mean pressure, $V$ is the velocity of bearing surface, $\sigma$ is the standard deviation (SD) of random surface roughness, $\varphi_{\mathrm{x}}$ and $\varphi_{\mathrm{y}}$ are pressure-flow factors and can be thought of as correction factors for the mean pressure flow in a rough bearing to that of the smooth bearing having the same nominal geometry, $\varphi_{\mathrm{s}}$ is the shearing flow factor, which relates the combined effect of the roughness and sliding in the net mean flow.

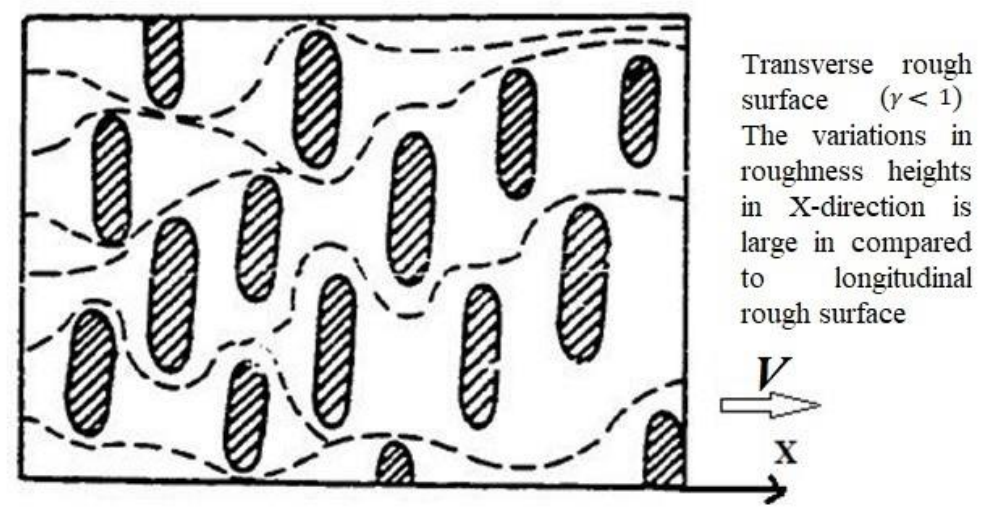

Fig. 1. Transverse rough surface [14]. 
Here the flow is presumed to be one-dimensional and steady in X-direction. Since the surface roughness is transverse $(\gamma<1)$, the variations in roughness heights in X-direction is significant (Fig. 1), so the effect of $\varphi_{s}$ maybe treated as noteworthy and cannot be neglected as in the case of the rough longitudinal surface.

Therefore, Eq. (1) is reduced to,

$$
\frac{d}{d x}\left[\varphi_{x} \frac{b_{T}^{3}}{12 \eta} \frac{d \bar{p}}{d x}\right]=\frac{V}{2} \frac{d \overline{b_{T}}}{d x}+\frac{V \sigma}{2} \frac{d \varphi_{S}}{d x}
$$

Patir [14] described the estimation of the average film thickness (mean gap) by,

$$
\overline{b_{T}}=\int_{-c}^{c}(b+\delta) f(\delta) d \delta=E(b)
$$

Where,

$$
b_{\mathrm{T}}=b+\delta
$$

The geometry of PSB is shown in Fig. 2

$$
b(x)=b_{0}+\left(b_{1}-b_{0}\right)\left(1-\frac{x}{l}\right)
$$

The geometry of ESB is shown in Fig. 3

$$
b(x)=b_{1} \operatorname{Exp}\left(-\frac{x}{l} \ln \frac{b_{1}}{b_{0}}\right)
$$

The geometry of HSB is shown in Fig. 3

$$
b(x)=b_{1}\left[1+\frac{x}{l}\left(\frac{b_{1}}{b_{0}}-1\right)\right]^{-1}
$$

The geometry of SSB is shown in Fig. 3

$$
b(x)=b_{0} \sec \left[\left(1-\frac{x}{l}\right) \sec ^{-1}\left(\frac{b_{1}}{b_{0}}\right)\right]
$$

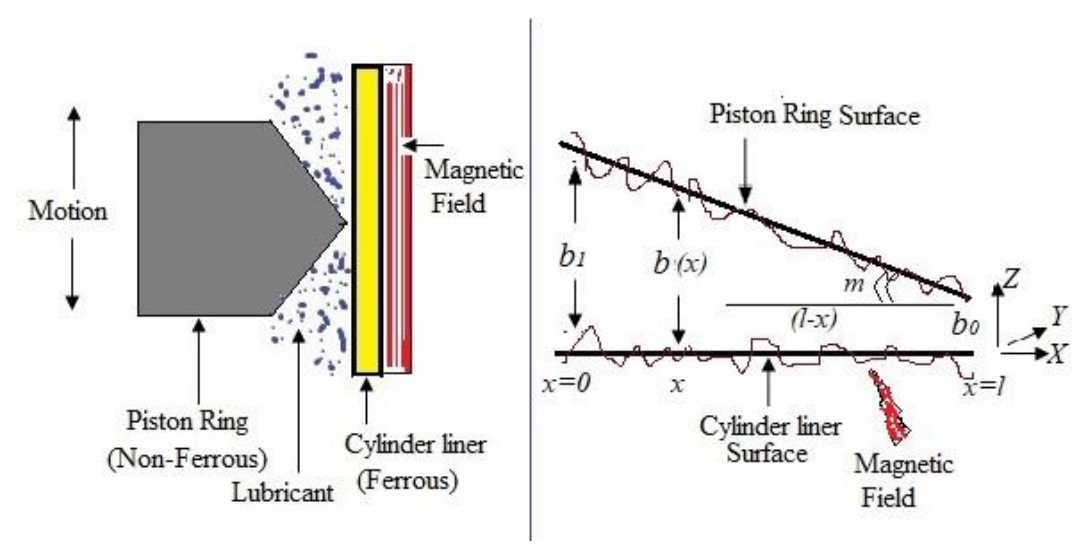

Fig. 2. The geometry of PSB and piston ring-cylinder assembly [7]. 


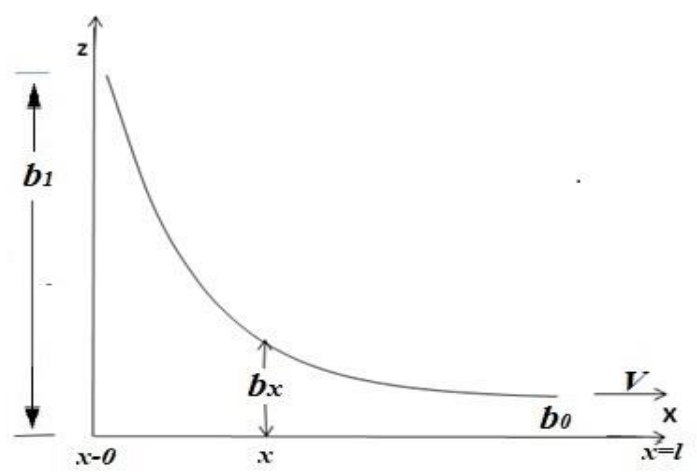

Fig. 3. Approximate geometry of HSB, ESB, and SSB [6].

We use the magnetic fluid (Ferro-fluid) instead of any regular lubricant and an external magnetic field produced by an electromagnet or permanent magnet to magnetize the Ferro-fluid. Such magnets can be fixed around the cylinder surface of the piston-ring assembly of an IC engine. Many engineering applications, such as machine tools, gears, sliding contact bearings, clutch plates, etc., use this kind of magnetism.

Let the magnetic field $M$ be applied to diminish wear on the sliding material and get better LTC at the required contact zone. Since the flow is considered in X-direction only, we have,

$$
\frac{\partial}{\partial y}\left(\bar{p}-0.5 \mu_{0} \mu_{f} M^{2}\right)=0
$$

Therefore, $M^{2}$ should be a function of $x$. i.e., $M^{2}=M^{2}(x)$.

We now can write the components of the applied magnetic field $M=\left(M_{x}, M_{y}, M_{z}\right)$ as

$$
M_{x}=M(x) \cos \theta, \quad M_{y}=M(x) \sin \theta, \quad M_{z}=0
$$

and $M^{2}=M \cdot M=M_{x}^{2}+M_{y}^{2}+M_{z}^{2}=M^{2}(x)$

Here we assume that $M^{2}$ should obey the condition,

$$
M^{2}=0 \text { at } x=0 \text { and } x=l
$$

So, the applied magnetic field $M$ is assumed to be of magnitude $M^{2}=x(l-x)$ slanting to the stable surface of the slider bearing [16]. Here it is chosen in such a way to balance the dimensions of both sides.

As an outcome of this application, bearing maintenance cost reduces to a substantial extent. The pressure at the contact zone is assumed to be increased concerning the applied magnetism. Hence the Eq. (2) can be modified under the usual assumptions of the hydrodynamic lubrication [15-16] as:

$$
\frac{d}{d x}\left[\varphi_{x} \frac{b_{T}^{3}}{12 \eta} \frac{d}{d x}\left(\bar{p}-0.5 \mu_{0} \mu_{f} M^{2}\right)\right]=\frac{V}{2} \frac{d \overline{b_{T}}}{d x}+\frac{V \sigma}{2} \frac{d \varphi_{S}}{d x}
$$

Since the roughness amplitude- $\delta$ in the surface roughness is a random variable, we use the probability density function $f(\delta)$ to introduce the mean, variance, and skewness of the surface roughness in the form of expected values [15] as $\alpha=E(\rho), \sigma^{2}=E\left[(\rho-\alpha)^{2}\right]$, and $\varepsilon=E\left[(\rho-\alpha)^{3}\right]$ respectively. 
Where the expectancy operator- $E(\bullet)$ represents the expected value of $(\bullet)$ and is defined as $E(\bullet)=\int_{-c}^{c}(\bullet) f(\delta) d \delta$.

Averaging the Eq. (7), it will turn into,

$$
\frac{d}{d x}\left[\varphi_{x} \frac{g_{b}}{12 \eta} \frac{d}{d x}\left\{(\bar{p})-0.5 \mu_{0} \mu_{f} M^{2}\right\}\right]=\frac{V}{2} \frac{d \bar{b}}{d x}+\frac{V \sigma}{2} \frac{d \varphi_{s}}{d x}
$$

Where

$$
\begin{aligned}
& g_{b}=E\left(b_{T}{ }^{3}\right)=E\left((b+\delta)^{3}\right) \\
& =\int_{-c}^{c}(b+\delta)^{3} f(\delta) d \delta \\
& =\int_{-c}^{c}\left(b^{3}+3 b^{2} \delta+3 b \delta^{2}+\delta^{3}\right) f(\delta) d \delta \\
& =b^{3} \int_{-c}^{c} f(\delta) d \delta+3 b^{2} \int_{-c}^{c} \delta f(\delta) d \delta+3 b \int_{-c}^{c} \delta^{2} f(\delta) d \delta+\int_{-c}^{c} \delta^{3} f(\delta) d \delta \\
& =b^{3}+3 b^{2}(\alpha)+3 b\left(\sigma^{2}+\alpha^{2}\right)+\left(3 \sigma^{2} \alpha+\alpha^{3}+\varepsilon\right)
\end{aligned}
$$

Introducing the dimensionless quantities,

$$
\begin{aligned}
& b^{*}=\frac{b}{b_{0}}, X=\frac{x}{l}, P^{*}=\frac{b_{0}^{2}(\bar{p})}{\eta V l} \\
& G_{b}^{*}=b^{* 3}+3 b^{* 2}\left(\alpha^{*}\right)+3 b^{*}\left(\alpha^{* 2}+\sigma^{* 2}\right)+\left(3 \sigma^{* 2} \alpha^{*}+\alpha^{* 3}+\varepsilon^{*}\right)
\end{aligned}
$$

We have the non-dimensional form of Eq. (8) as,

$$
\frac{d}{d X}\left[\varphi_{X} G_{b}^{*} \frac{d}{d X}\left\{P^{*}-\mu^{*} X(1-X)\right\}\right]=6 \frac{d}{d X}\left[b^{*}+\sigma^{*} \phi_{s}\right]
$$

Where the experimental relations for $\varphi_{x}$ and $\varphi_{s}$ are as under [14],

$$
\begin{array}{ll}
\varphi_{x}=1-C e^{\{-r H\}}=1+C e^{\left\{-r b^{*} B_{0}\right\}} & (\text { for } \gamma<1) \\
\phi_{S}=A_{1}\left(b^{*} B\right)^{\alpha_{1}} e^{-\alpha_{2}\left(b^{*} B_{0}\right)+\alpha_{3}\left(b^{*} B_{0}\right)^{2}} & (\text { for } \gamma<1)
\end{array}
$$

Where

$$
B=\frac{b}{\sigma}, B_{0}=\frac{b_{0}}{\sigma}, B_{m}=\frac{b_{0}}{\sigma}
$$

The constants $r, C, A_{1}, \alpha_{1}, \alpha_{2}$, and $\alpha_{3}$ are expressed as a function of $\gamma$ (Tables 1 and 2 [14]) in case of the transverse-rough surface.

Table 1. Relation among $\gamma, \mathrm{C}, \mathrm{r}$, and B [14].

\begin{tabular}{llll}
\hline$\gamma$ & $C$ & $r$ & $B$ \\
\hline $1 / 3$ & 1.16 & 0.42 & $\mathrm{~B}>0.5$ \\
$1 / 6$ & 1.38 & 0.42 & $\mathrm{~B}>0.5$ \\
$1 / 9$ & 1.48 & 0.42 & $\mathrm{~B}>0.5$ \\
\hline
\end{tabular}

Table 2. Relation between $A_{l}, \alpha_{i}(i=1,2,3), \gamma$, and B [14].

\begin{tabular}{llllll}
\hline$\gamma$ & $A_{1}$ & $\alpha_{1}$ & $\alpha_{2}$ & $\alpha_{3}$ & $B$ \\
\hline $1 / 3$ & 1.858 & 1.01 & 0.76 & 0.03 & $\mathrm{~B}>0.5$ \\
$1 / 6$ & 1.962 & 1.08 & 0.77 & 0.03 & $\mathrm{~B}>0.5$ \\
$1 / 9$ & 2.046 & 1.12 & 0.78 & 0.03 & $\mathrm{~B}>0.5$ \\
\hline
\end{tabular}


Assuming the B. C.: $P^{*}=0$ at $X=0,1$, and $\frac{d P^{*}}{d X}=0$, where the mean gap between two surfaces is maximum, Eq. (9) can be turned out to be,

$$
P^{*}(X)=\int_{0}^{X} \frac{1}{\varphi_{X} G_{b}^{*}}\left[6\left(b^{*}+\sigma^{*} \phi_{s}\right)-K^{*}\right] d X
$$

Where,

$$
K^{*}=\frac{\int_{0}^{1} \frac{6\left(b^{*}+\sigma^{*} \phi_{s}\right)}{\varphi_{X} G_{b}^{*}} d X}{\int_{0}^{1} \frac{1}{\varphi_{X} G_{b}^{*}} d X}
$$

The dimensionless LTC $\left(W^{*}\right)$ per unit width is,

$$
\begin{aligned}
& W^{*}=\frac{w \cdot b_{0}^{2}}{\mu V l}=\int_{0}^{1} P^{*} d X \\
& \therefore W^{*}=\int_{0}^{1}\left[\int_{0}^{X} \frac{1}{\varphi_{X} G_{b}^{*}}\left[6\left(b^{*}+\sigma^{*} \phi_{S}\right)-K^{*}\right] d X\right] d X
\end{aligned}
$$

\section{Results and Discussion}

The LTC in dimensionless form is attained by Eq. (11). Here the integrals throughout the mathematical analysis to get the LTC explicitly are evaluated by Simpson's 1/3-rule by taking step size of 0.1 for $\mathrm{X}=0$ to 1 , and graphs present the results.

Figs. 4-19 compare the behavior of the dimensionless value LTC of the corresponding bearings concerning different parameters, like mean, SD, skewness, RPP, and magnetism. Observing these results, we can see that the dimensionless LTC's highest value is obtained in the ESB while the PSB produces the least LTC.

In the PSB, Figs. 4,8,12,16 show that the highest value of dimensionless LTC is approximately 0.60 by fixing other parameters as $\alpha^{*}=-0.5, \sigma^{*}=0.1, \varepsilon^{*}=-0.025$, and $\gamma=1 / 6$. The LTC increases around 0.59 to 0.61 while the RPP $(\gamma)$ moves from $1 / 3$ to $1 / 9$. That

\begin{tabular}{|c|c|c|c|c|c|c|}
\hline \multicolumn{3}{|c|}{ LTC vs $\left(\mu^{*} \& \alpha^{*}\right)$} & \multicolumn{3}{|c|}{$\left(\gamma=1 / 6, \sigma^{*}=0.1, \varepsilon^{*}=-0.025\right)-\mathrm{PSB}$} & \\
\hline$\mu^{*}$ & 0.02 & 0.04 & 0.06 & 0.08 & 0.1 & \\
\hline \multirow{5}{*}{ LTC } & 0.479009 & 0.511009 & 0.543009 & 0.575009 & 0.607009 & $\alpha^{*}=-0.05$ \\
\hline & 0.463847 & 0.495847 & 0.527847 & 0.559847 & 0.591847 & $\alpha^{*}=-0.025$ \\
\hline & 0.449391 & 0.481391 & 0.513391 & 0.545391 & 0.577391 & $\alpha^{*}=0$ \\
\hline & 0.435598 & 0.467598 & 0.499598 & 0.531598 & 0.563598 & $\alpha^{*}=0.025$ \\
\hline & 0.422428 & 0.454428 & 0.486428 & 0.518428 & 0.550428 & $\alpha^{*}=0.05$ \\
\hline
\end{tabular}
means that the LTC is higher in the case when the surface is more transverse. The numerical comparisons are given in the following Tables 3-6.

Table 3. LTC vs $\left(\mu^{*} \& \alpha^{*}\right)\left(\gamma=1 / 6, \sigma^{*}=0.1, \varepsilon^{*}=-0.025\right)-$ PSB. 
Table 4. LTC vs $\left(\mu^{*} \& \sigma^{*}\right)\left(\gamma=1 / 6, \alpha^{*}=-0.05, \varepsilon^{*}=-0.025\right)-$ PSB.

\begin{tabular}{|c|c|c|c|c|c|c|}
\hline \multicolumn{7}{|c|}{ LTC vs $\left(\mu^{*} \& \sigma^{*}\right) \quad\left(\gamma=1 / 6, \alpha^{*}=-0.05, \varepsilon^{*}=-0.025\right)-$ PSB } \\
\hline$\mu^{*}$ & 0.02 & 0.04 & 0.06 & 0.08 & 0.1 & \\
\hline \multirow{5}{*}{ LTC } & 0.495531 & 0.527531 & 0.559531 & 0.591531 & 0.623531 & $\sigma^{*}=0$ \\
\hline & 0.492126 & 0.524126 & 0.556126 & 0.588126 & 0.620126 & $\sigma^{*}=0.025$ \\
\hline & 0.488222 & 0.520222 & 0.552222 & 0.584222 & 0.616222 & $\sigma^{*}=0.05$ \\
\hline & 0.483841 & 0.515841 & 0.547841 & 0.579841 & 0.611841 & $\sigma^{*}=0.075$ \\
\hline & 0.479009 & 0.511009 & 0.543009 & 0.575009 & 0.607009 & $\sigma^{*}=0.1$ \\
\hline
\end{tabular}

Table 5. LTC vs $\left(\mu^{*} \& \varepsilon^{*}\right)\left(\gamma=1 / 6, \alpha^{*}=-0.05, \sigma^{*}=0.1\right)-$ PSB.

\begin{tabular}{lllllll}
\hline \multicolumn{6}{c}{ LTC vs $\left(\mu^{*} \& \varepsilon^{*}\right)\left(\gamma=1 / 6, \alpha^{*}=-0.05, \sigma^{*}=0.1\right)-$ PSB } \\
\hline$\mu^{*}$ & 0.02 & 0.04 & 0.06 & 0.08 & 0.1 & \\
\hline & 0.479009 & 0.511009 & 0.543009 & 0.575009 & $\mathbf{0 . 6 0 7 0 0 9}$ & $\varepsilon^{*=}=0.025$ \\
& 0.477474 & 0.509474 & 0.541474 & 0.573474 & 0.605474 & $\varepsilon^{*=}=0.01$ \\
LTC & 0.476463 & 0.508463 & 0.540463 & 0.572463 & 0.604463 & $\varepsilon^{*=0}$ \\
& 0.475463 & 0.507463 & 0.539463 & 0.571463 & 0.603463 & $\varepsilon^{*=0} 01$ \\
& 0.473981 & 0.505981 & 0.537981 & 0.569981 & 0.601981 & $\varepsilon^{*=}=0.025$ \\
\hline
\end{tabular}

Table 6. LTC vs $\left(\mu^{*} \& \gamma\right)\left(\alpha^{*}=-0.05, \sigma^{*}=0.1, \varepsilon^{*=-0} 0.025\right)-$ PSB.

\begin{tabular}{|c|c|c|c|c|c|c|}
\hline \multicolumn{7}{|c|}{ LTC vs $\left(\mu^{*} \& \gamma\right) \quad\left(\alpha^{*}=-0.05, \sigma^{*}=0.1, \varepsilon^{*=-0.025}\right)-$ PSB } \\
\hline$\mu^{*}$ & 0.02 & 0.04 & 0.06 & 0.08 & 0.1 & \\
\hline \multirow{3}{*}{ LTC } & 0.471241 & 0.503241 & 0.535241 & 0.567241 & 0.599241 & $\gamma=1 / 3$ \\
\hline & 0.479009 & 0.511009 & 0.543009 & 0.575009 & 0.607009 & $\gamma=1 / 6$ \\
\hline & 0.482887 & 0.514887 & 0.546887 & 0.578887 & 0.610887 & $\gamma=1 / 9$ \\
\hline
\end{tabular}

In the HSB, Figures 5,9,13,17 show the highest value of dimensionless LTC is approximately 0.82 by fixing other parameters as $\alpha^{*}=-0.5, \sigma^{*}=0.1, \varepsilon^{*}=-0.025$, and $\gamma=1 / 6$. The numerical comparisons are given in the following Tables 7-10.

Table 7. LTC vs $\left(\mu^{*} \& \alpha^{*}\right)\left(\gamma=1 / 6, \sigma^{*}=0.1, \varepsilon^{*}=-0.025\right)-$ HSB.

\begin{tabular}{lllllll}
\hline \multicolumn{7}{c}{ LTC vs $\left(\mu^{*} \& \alpha^{*}\right)\left(\gamma=1 / 6, \sigma^{*}=0.1, \varepsilon^{*}=-0.025\right)-$ HSB } \\
\hline$\mu^{*}$ & 0.02 & 0.04 & 0.06 & 0.08 & 0.1 & \\
\hline \multirow{4}{*}{ LTC } & 0.696528 & 0.728528 & 0.760528 & 0.792528 & 0.824528 & $\alpha^{*}=-0.05$ \\
& 0.658032 & 0.690032 & 0.722032 & 0.754032 & 0.786032 & $\alpha^{*}=-0.025$ \\
& 0.622512 & 0.654512 & 0.686512 & 0.718512 & 0.750512 & $\alpha^{*}=0$ \\
& 0.589681 & 0.621681 & 0.653681 & 0.685681 & 0.717681 & $\alpha^{*}=0.025$ \\
& 0.559283 & 0.591283 & 0.623283 & 0.655283 & 0.687283 & $\alpha^{*}=0.05$ \\
\hline
\end{tabular}

Table 8. LTC vs $\left(\mu^{*} \& \alpha^{*}\right)\left(\gamma=1 / 6, \sigma^{*}=0.1, \varepsilon^{*=-0.025)}-\right.$ HSB.

\begin{tabular}{|c|c|c|c|c|c|c|}
\hline \multicolumn{7}{|c|}{ LTC vs $\left(\mu^{*} \& \sigma^{*}\right)\left(\gamma=1 / 6, \alpha^{*}=-0.05, \varepsilon^{*}=-0.025\right)-$ HSB } \\
\hline$\mu^{*}$ & 0.02 & 0.04 & 0.06 & 0.08 & 0.1 & \\
\hline \multirow{5}{*}{ LTC } & 0.746979 & 0.778979 & 0.810979 & 0.842979 & 0.874979 & $\sigma^{*}=0$ \\
\hline & 0.736911 & 0.768911 & 0.800911 & 0.832911 & 0.864911 & $\sigma^{*}=0.025$ \\
\hline & 0.725064 & 0.757064 & 0.789064 & 0.821064 & 0.853064 & $\sigma^{*}=0.05$ \\
\hline & 0.711555 & 0.743555 & 0.775555 & 0.807555 & 0.839555 & $\sigma^{*}=0.075$ \\
\hline & 0.696528 & 0.728528 & 0.760528 & 0.792528 & 0.824528 & $\sigma^{*}=0.1$ \\
\hline
\end{tabular}


Table 9. LTC vs $\left(\mu^{*} \& \varepsilon^{*}\right)\left(\gamma=1 / 6, \alpha^{*}=-0.05, \sigma^{*}=0.1\right)-$ HSB.

\begin{tabular}{lllllll}
\hline \multicolumn{6}{c}{ LTC vs $\left(\mu^{*} \& \varepsilon^{*}\right)\left(\gamma=1 / 6, \alpha^{*}=-0.05, \sigma^{*}=0.1\right)-$ HSB } \\
\hline$\mu^{*}$ & 0.02 & 0.04 & 0.06 & 0.08 & 0.1 & \\
\hline \multirow{4}{*}{ LTC } & 0.696528 & 0.728528 & 0.760528 & 0.792528 & 0.824528 & $\varepsilon^{*=-0.025}$ \\
& 0.690528 & 0.722528 & 0.754528 & 0.786528 & 0.818528 & $\varepsilon^{*=-0} 0.01$ \\
& 0.686598 & 0.718598 & 0.750598 & 0.782598 & 0.814598 & $\varepsilon^{*=0}$ \\
& 0.682723 & 0.714723 & 0.746723 & 0.778723 & 0.810723 & $\varepsilon^{*=0} 0.01$ \\
& 0.67701 & 0.70901 & 0.74101 & 0.77301 & 0.80501 & $\varepsilon^{*=0} 0.025$ \\
\hline
\end{tabular}

Table 10. LTC vs $\left(\mu^{*} \& \gamma\right)\left(\alpha^{*}=-0.05, \sigma^{*}=0.1, \varepsilon^{*}=-0.025\right)-$ HSB.

\begin{tabular}{|c|c|c|c|c|c|c|}
\hline & \multicolumn{2}{|c|}{ LTC vs $\left(\mu^{*} \& \gamma\right)$} & \multicolumn{3}{|c|}{$\left(\alpha^{*}=-0.05, \sigma^{*}=0.1, \varepsilon^{*}=-0.025\right)-\mathrm{HSB}$} & \\
\hline$\mu^{*}$ & 0.02 & 0.04 & 0.06 & 0.08 & 0.1 & \\
\hline \multirow{3}{*}{ LTC } & 0.663886 & 0.695886 & 0.727886 & 0.759886 & 0.791886 & $\gamma=1 / 3$ \\
\hline & 0.696528 & 0.728528 & 0.760528 & 0.792528 & 0.824528 & $\gamma=1 / 6$ \\
\hline & 0.713733 & 0.745733 & 0.777733 & 0.809733 & 0.841733 & $\gamma=1 / 9$ \\
\hline
\end{tabular}

In the ESB, Figs. 6,10,14,18 show that the highest value of dimensionless LTC is approximately 0.84 by fixing other parameters as $\alpha^{*}=-0.5, \sigma^{*}=0.1, \varepsilon^{*}=-0.025$, and $\gamma=1 / 6$. The LTC increases from 0.80 to 0.85 while the RPP $(\gamma)$ moves from $1 / 3$ to $1 / 9$. In this case, it also reveals that the LTC is higher when the surface is more transverse. The numerical comparisons are given in the following Tables 11-14.

Table 11. LTC vs $\left(\mu^{*} \& \alpha^{*}\right)\left(\gamma=1 / 6, \sigma^{*}=0.1, \varepsilon^{*}=-0.025\right)-$ ESB.

\begin{tabular}{lllllll}
\hline \multicolumn{6}{c}{ LTC vs $\left(\mu^{*} \& \alpha^{*}\right)$} & $\left(\gamma=1 / 6, \sigma^{*}=0.1, \varepsilon^{*=-} 0.025\right)-$ ESB \\
\hline$\mu^{*}$ & 0.02 & 0.04 & 0.06 & 0.08 & 0.1 & \\
\hline \multirow{4}{*}{ LTC } & 0.71669 & 0.74869 & 0.78069 & 0.81269 & 0.84469 & $\alpha^{*=-0.05}$ \\
& 0.6779 & 0.7099 & 0.7419 & 0.7739 & 0.8059 & $\alpha^{*=-0.025}$ \\
& 0.64203 & 0.67403 & 0.70603 & 0.73803 & 0.77003 & $\alpha^{*=0}$ \\
& 0.608807 & 0.640807 & 0.672807 & 0.704807 & 0.736807 & $\alpha^{*}=0.025$ \\
& 0.577988 & 0.609988 & 0.641988 & 0.673988 & 0.705988 & $\alpha^{*}=0.05$
\end{tabular}

Table 12. LTC vs $\left(\mu^{*} \& \sigma^{*}\right)\left(\gamma=1 / 6, \alpha^{*}=-0.05, \varepsilon^{*}=-0.025\right)-$ ESB.

\begin{tabular}{|c|c|c|c|c|c|c|}
\hline \multicolumn{7}{|c|}{ LTC vs $\left(\mu^{*} \& \sigma^{*}\right)\left(\gamma=1 / 6, \alpha^{*}=-0.05, \varepsilon^{*}=-0.025\right)-$ ESB } \\
\hline$\mu^{*}$ & 0.02 & 0.04 & 0.06 & 0.08 & 0.1 & \\
\hline \multirow{5}{*}{ LTC } & 0.767182 & 0.799182 & 0.831182 & 0.863182 & 0.895182 & $\sigma^{*}=0$ \\
\hline & 0.757068 & 0.789068 & 0.821068 & 0.853068 & 0.885068 & $\sigma^{*}=0.025$ \\
\hline & 0.745203 & 0.777203 & 0.809203 & 0.841203 & 0.873203 & $\sigma^{*}=0.05$ \\
\hline & 0.731698 & 0.763698 & 0.795698 & 0.827698 & 0.859698 & $\sigma^{*}=0.075$ \\
\hline & 0.71669 & 0.74869 & 0.78069 & 0.81269 & 0.84469 & $\sigma^{*}=0.1$ \\
\hline
\end{tabular}

Table 13. LTC vs $\left(\mu^{*} \& \varepsilon^{*}\right)\left(\gamma=1 / 6, \alpha^{*=-0} 0.05, \sigma^{*}=0.1\right)-$ ESB.

\begin{tabular}{lllllll}
\hline \multicolumn{6}{c}{ LTC vs $\left(\mu^{*} \& \varepsilon^{*}\right)$} & $\left(\gamma=1 / 6, \alpha^{*}=-0.05, \sigma^{*}=0.1\right)-$ ESB \\
\hline$\mu^{*}$ & 0.02 & 0.04 & 0.06 & 0.08 & 0.1 & \\
\multirow{2}{*}{ LTC } & 0.71669 & 0.74869 & 0.78069 & 0.81269 & 0.84469 & $\varepsilon^{*=-0.025}$ \\
& 0.710868 & 0.742868 & 0.774868 & 0.806868 & 0.838868 & $\varepsilon^{*=-0.01}$ \\
\hline
\end{tabular}




\begin{tabular}{|c|c|c|c|c|c|c|}
\hline \multicolumn{7}{|c|}{ LTC vs $\left(\mu^{*} \& \varepsilon^{*}\right) \quad\left(\gamma=1 / 6, \alpha^{*}=-0.05, \sigma^{*}=0.1\right)-$ ESB } \\
\hline$\mu^{*}$ & 0.02 & 0.04 & 0.06 & 0.08 & 0.1 & \\
\hline & 0.70705 & 0.73905 & 0.77105 & 0.80305 & 0.83505 & $\varepsilon^{*}=0$ \\
\hline & 0.703283 & 0.735283 & 0.767283 & 0.799283 & 0.831283 & $\varepsilon^{*}=0.01$ \\
\hline & 0.697723 & 0.729723 & 0.761723 & 0.793723 & 0.825723 & $\varepsilon^{*}=0.025$ \\
\hline
\end{tabular}

Table 14. LTC vs $\left(\mu^{*} \& \gamma\right)\left(\alpha^{*}=-0.05, \sigma^{*}=0.1, \varepsilon^{*}=-0.025\right)-$ ESB.

\begin{tabular}{lllllll}
\hline \multicolumn{7}{c}{ LTC vs $\left(\mu^{*} \& \gamma\right)\left(\alpha^{*}=-0.05, \sigma^{*}=0.1, \varepsilon^{*}=-0.025\right)-$ ESB } \\
\hline$\mu^{*}$ & 0.02 & 0.04 & 0.06 & 0.08 & 0.1 & \\
\hline \multirow{3}{*}{ LTC } & 0.684657 & 0.709416 & 0.741416 & 0.773416 & 0.805416 & $\gamma=1 / 3$ \\
& 0.71669 & 0.740944 & 0.772944 & 0.804944 & 0.836944 & $\gamma=1 / 6$ \\
& 0.733482 & 0.757464 & 0.789464 & 0.821464 & 0.853464 & $\gamma=1 / 9$ \\
\hline
\end{tabular}

In the SSB, Figs. 7,11,15,19 show that the highest value of dimensionless LTC is approximately 0.73 by fixing other parameters as $\alpha^{*}=-0.5, \sigma^{*}=0.1, \varepsilon^{*}=-0.025$, and $\gamma=1 / 6$. The numerical comparisons are given in the following Tables 15-18.

Table 15. LTC vs $\left(\mu^{*} \& \alpha^{*}\right)\left(\gamma=1 / 6, \sigma^{*}=0.1, \varepsilon^{*}=-0.025\right)-$ SSB.

\begin{tabular}{|c|c|c|c|c|c|c|}
\hline \multicolumn{7}{|c|}{ LTC vs $\left(\mu^{*} \& \alpha^{*}\right) \quad\left(\gamma=1 / 6, \sigma^{*}=0.1, \varepsilon^{*=-0.025)}-\mathrm{SSB}\right.$} \\
\hline$\mu^{*}$ & 0.02 & 0.04 & 0.06 & 0.08 & 0.1 & \\
\hline \multirow{5}{*}{ LTC } & 0.609449 & 0.641449 & 0.673449 & 0.705449 & 0.737449 & $\alpha^{*}=-0.05$ \\
\hline & 0.574751 & 0.606751 & 0.638751 & 0.670751 & 0.702751 & $\alpha^{*}=-0.025$ \\
\hline & 0.542871 & 0.574871 & 0.606871 & 0.638871 & 0.670871 & $\alpha^{*}=0$ \\
\hline & 0.513521 & 0.545521 & 0.577521 & 0.609521 & 0.641521 & $\alpha^{*}=0.025$ \\
\hline & 0.486447 & 0.518447 & 0.550447 & 0.582447 & 0.614447 & $\alpha^{*}=0.05$ \\
\hline
\end{tabular}

Table 16. LTC vs $\left(\mu^{*} \& \sigma^{*}\right)\left(\gamma=1 / 6, \alpha^{*}=-0.05, \varepsilon^{*=-0.025}\right)-$ SSB.

\begin{tabular}{|c|c|c|c|c|c|c|}
\hline \multicolumn{7}{|c|}{ LTC vs $\left(\mu^{*} \& \sigma^{*}\right)\left(\gamma=1 / 6, \alpha^{*}=-0.05, \varepsilon^{*=-0.025}\right)-$ SSB } \\
\hline$\mu^{*}$ & 0.02 & 0.04 & 0.06 & 0.08 & 0.1 & \\
\hline \multirow{5}{*}{ LTC } & 0.65553 & 0.68753 & 0.71953 & 0.75153 & 0.78353 & $\sigma^{*}=0$ \\
\hline & 0.646404 & 0.678404 & 0.710404 & 0.742404 & 0.774404 & $\sigma^{*}=0.025$ \\
\hline & 0.635598 & 0.667598 & 0.699598 & 0.731598 & 0.763598 & $\sigma^{*}=0.05$ \\
\hline & 0.623231 & 0.655231 & 0.687231 & 0.719231 & 0.751231 & $\sigma^{*}=0.075$ \\
\hline & 0.609449 & 0.641449 & 0.673449 & 0.705449 & 0.737449 & $\sigma^{*}=0.1$ \\
\hline
\end{tabular}

Table 17. LTC vs $\left(\mu^{*} \& \varepsilon^{*}\right) \quad\left(\gamma=1 / 6, \alpha^{*}=-0.05, \sigma^{*}=0.1\right)-\operatorname{SSB}$.

\begin{tabular}{lllllll}
\hline \multicolumn{7}{c}{ LTC vS $\left(\mu^{*} \& \varepsilon^{*}\right)\left(\gamma=1 / 6, \alpha^{*}=-0.05, \sigma^{*}=0.1\right)-$ SSB } \\
\hline$\mu^{*}$ & 0.02 & 0.04 & 0.06 & 0.08 & 0.1 & \\
\hline \multirow{4}{*}{ LTC } & 0.609449 & 0.641449 & 0.673449 & 0.705449 & 0.737449 & $\varepsilon^{*=-0.025}$ \\
& 0.603588 & 0.635588 & 0.667588 & 0.699588 & 0.731588 & $\varepsilon^{*=-0.01}$ \\
& 0.599761 & 0.631761 & 0.663761 & 0.695761 & 0.727761 & $\varepsilon^{*=0}$ \\
& 0.595995 & 0.627995 & 0.659995 & 0.691995 & 0.723995 & $\varepsilon^{*=0} 0.01$ \\
& 0.590459 & 0.622459 & 0.654459 & 0.686459 & 0.718459 & $\varepsilon^{*=0} 0.025$ \\
\hline
\end{tabular}


Table 18. LTC vs $\left(\mu^{*} \& \gamma\right)\left(\alpha^{*=}=-0.05, \sigma^{*}=0.1, \varepsilon^{*=}=-0.025\right)-\mathrm{SSB}$.

\begin{tabular}{lllllll}
\hline \multicolumn{7}{c}{ LTC vs $\left(\mu^{*} \& \gamma\right)\left(\alpha^{*}=-0.05, \sigma^{*}=0.1, \varepsilon^{*}=-0.025\right)$} \\
\hline$\mu^{*}$ & 0.02 & 0.04 & 0.06 & 0.08 & 0.1 & \\
\hline \multirow{4}{*}{ LTC } & 0.578592 & 0.610592 & 0.642592 & 0.674592 & 0.706592 & $\gamma=1 / 3$ \\
& 0.609449 & 0.641449 & 0.673449 & 0.705449 & 0.737449 & $\gamma=1 / 6$ \\
& 0.625923 & 0.657923 & 0.689923 & 0.721923 & 0.753923 & $\gamma=1 / 9$ \\
\hline
\end{tabular}

Figs. 4-19 show that the LTC enhances by increasing the values of $\alpha^{*}(-\mathrm{ve}), \varepsilon^{*}(-\mathrm{ve})$, and $\mu^{*}$. The trends of LTC in all the figures with respect to $\alpha^{*}, \sigma^{*}, \varepsilon^{*}$, and $\gamma$ agree with the trends as obtained by Panchal et al. [7] which validates the results obtained in this article. Hence, the roughness parameters play an important role in improving the bearing's performance irrespective of its shape. The bearing's performance can be more enriched by giving the effect of the magnetic influence seen in the Gigures.

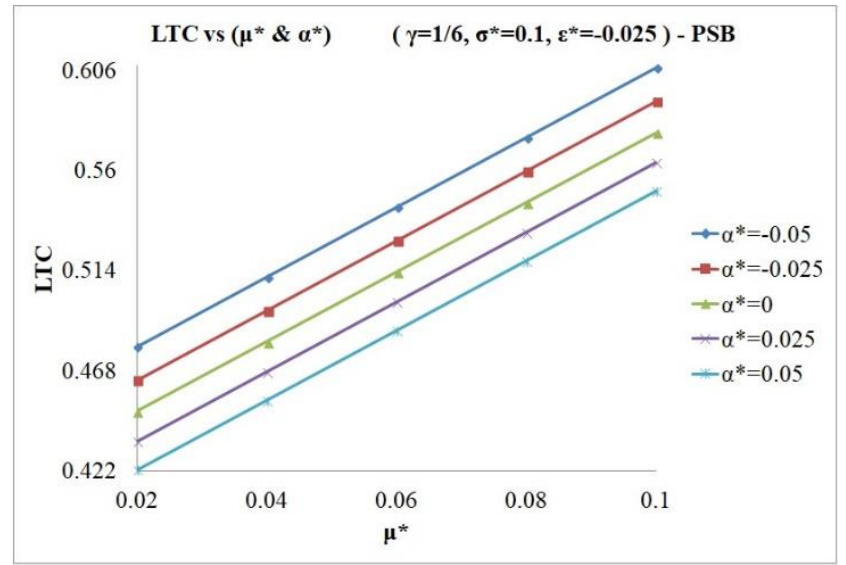

Fig. 4. LTC to ( $\mu^{*}$ and $\left.\alpha^{*}\right)$ : PSB.

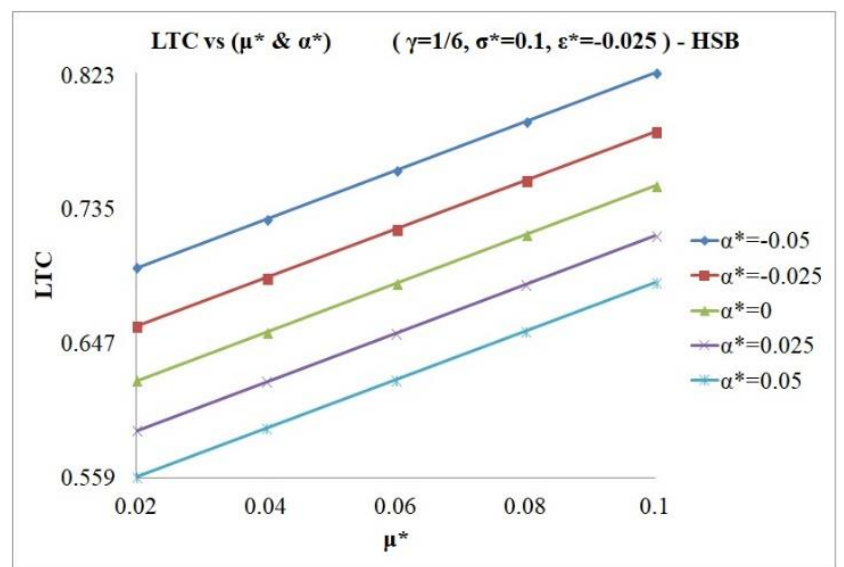

Fig. 5. LTC to ( $\mu^{*}$ and $\left.\alpha^{*}\right)$ : HSB. 


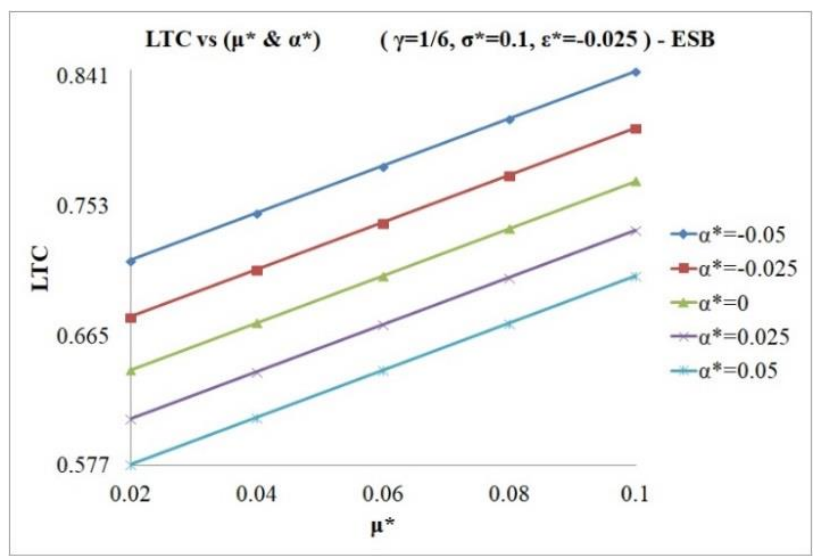

Fig. 6. LTC to ( $\mu^{*}$ and $\left.\alpha^{*}\right)$ : ESB.

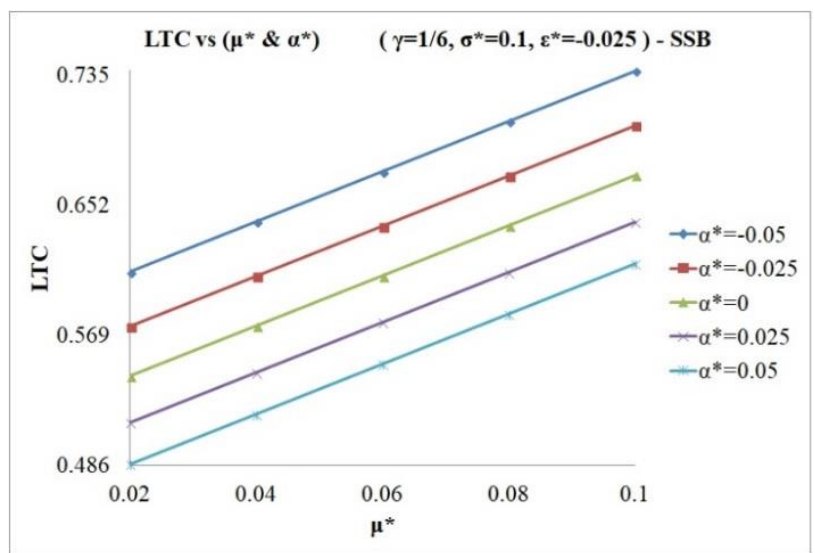

Fig. 7. LTC to ( $\mu^{*}$ and $\left.\alpha^{*}\right)$ : SSB.

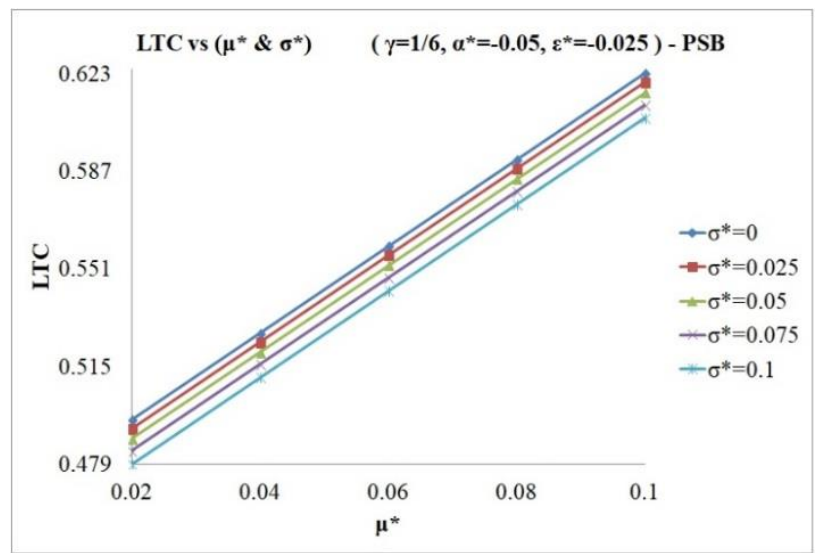

Fig. 8. LTC to ( $\mu^{*}$ and $\left.\sigma^{*}\right)$ : PSB. 


\section{Effect of Various Shapes of Rough Transverse Slider Bearing}

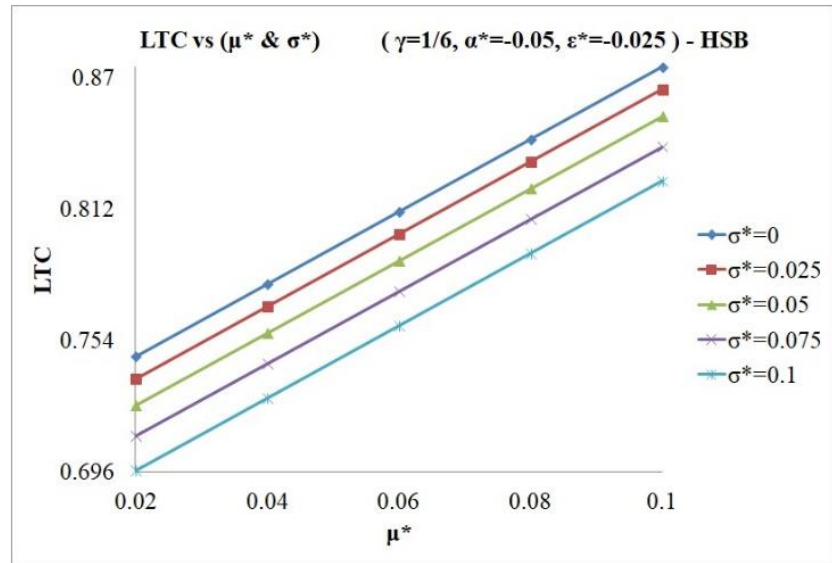

Fig. 9. LTC to ( $\mu^{*}$ and $\left.\sigma^{*}\right)$ : HSB.

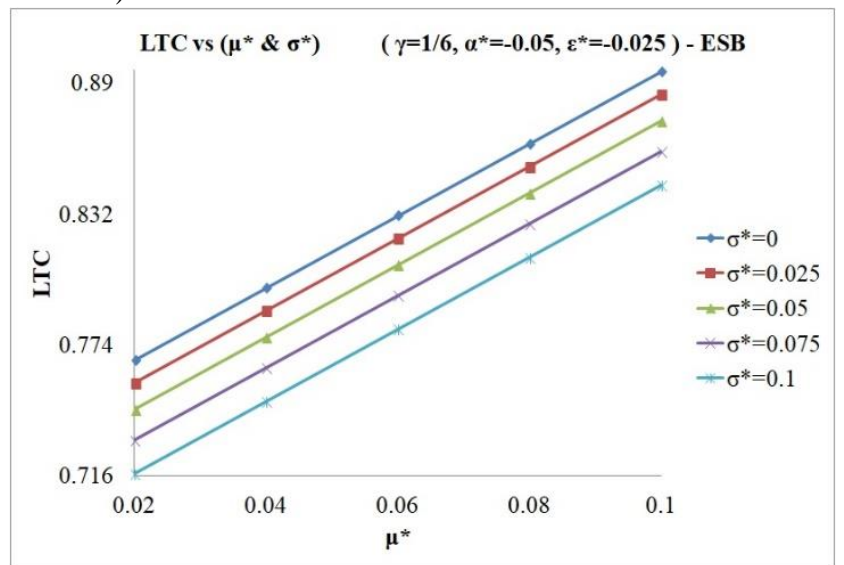

Fig. 10. LTC to $\left(\mu^{*}\right.$ and $\left.\sigma^{*}\right)$ : ESB.

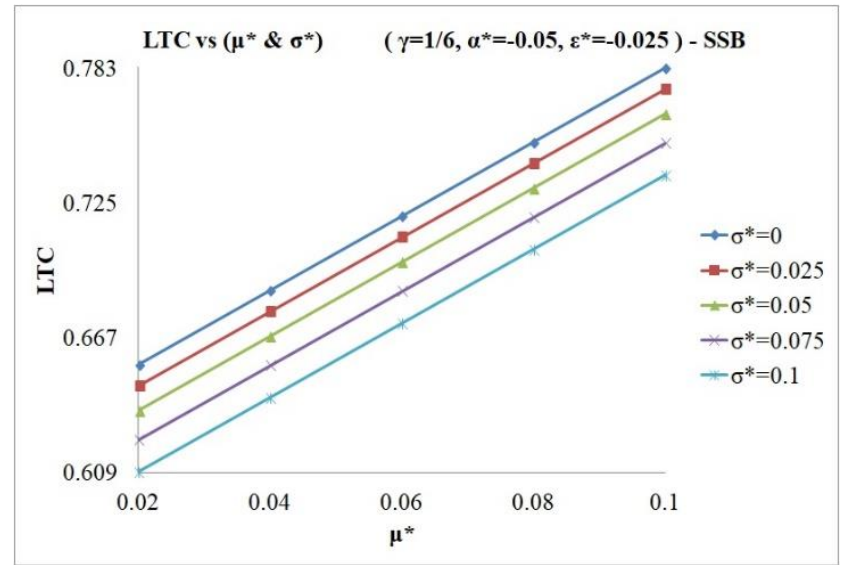

Fig. 11. LTC to $\left(\mu^{*}\right.$ and $\left.\sigma^{*}\right)$ : SSB. 


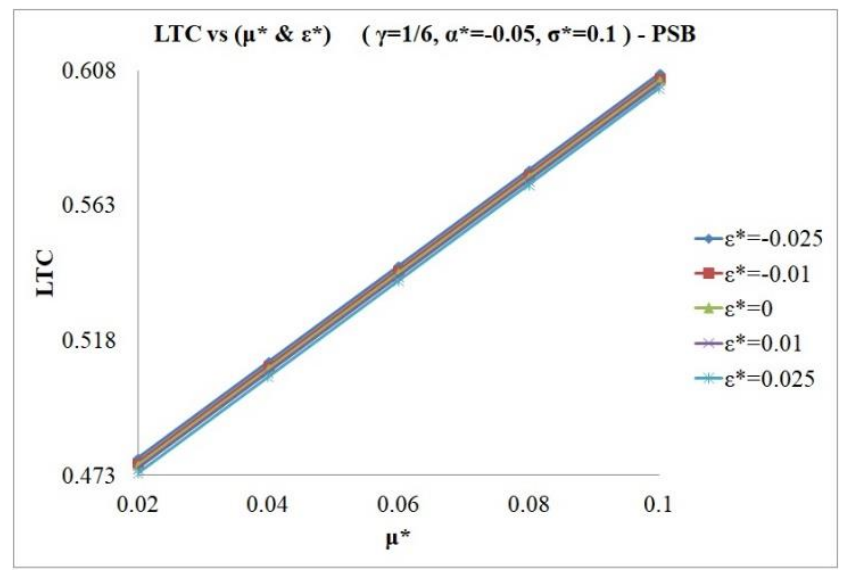

Fig. 12. LTC to ( $\mu^{*}$ and $\left.\varepsilon^{*}\right)$ : PSB.

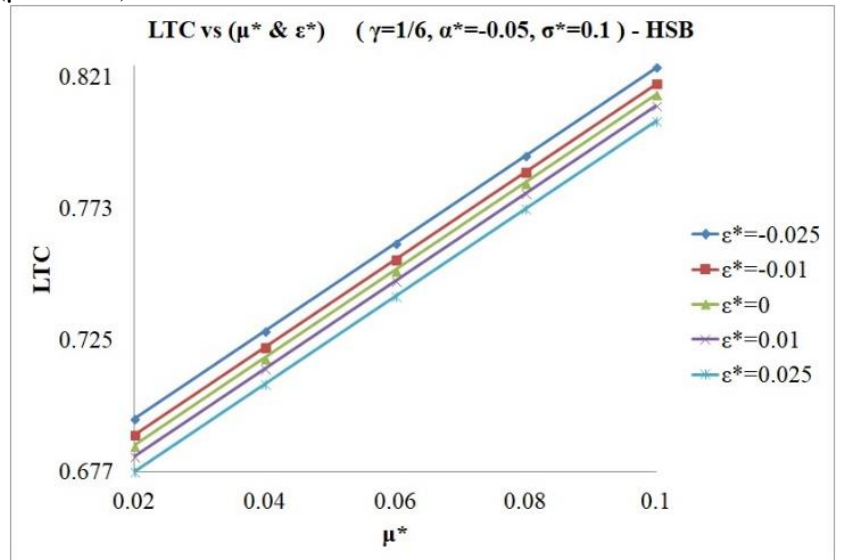

Fig. 13. LTC to $\left(\mu^{*}\right.$ and $\left.\varepsilon^{*}\right)$ : HSB.

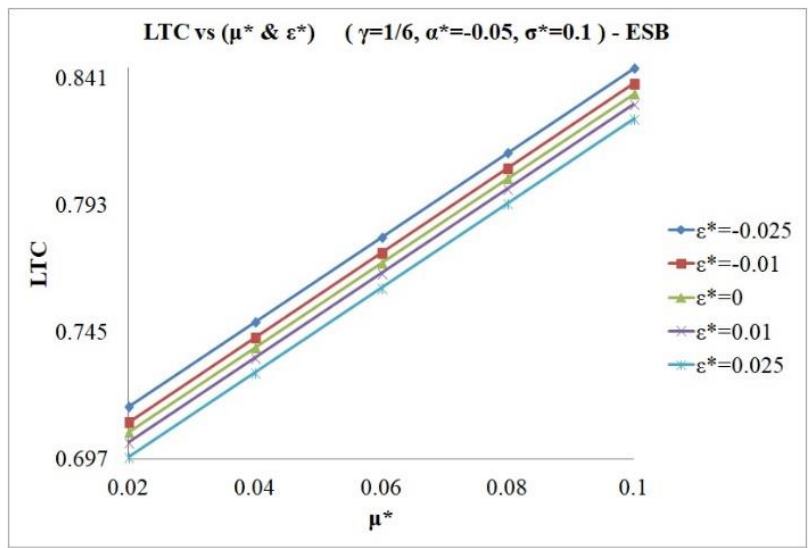

Fig. 14. LTC to ( $\mu^{*}$ and $\left.\varepsilon^{*}\right)$ : ESB. 


\section{Effect of Various Shapes of Rough Transverse Slider Bearing}

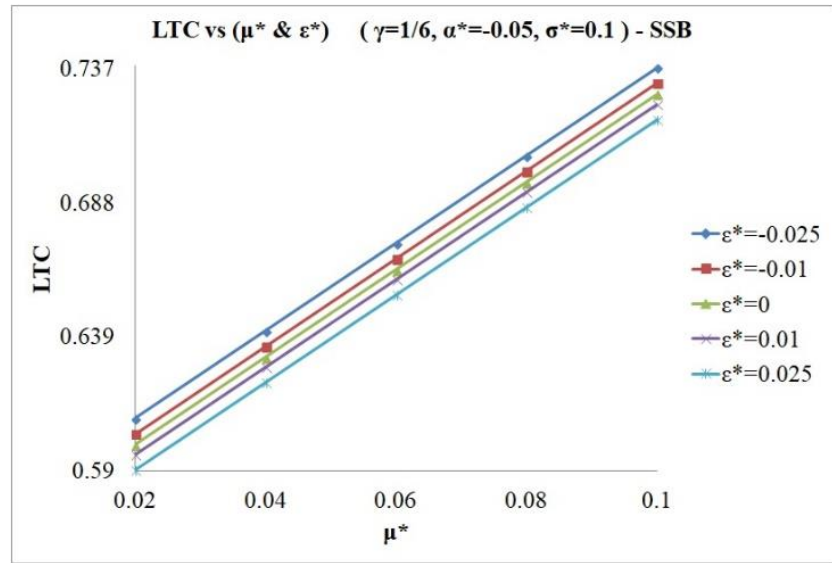

Fig. 15. LTC to $\left(\mu^{*}\right.$ and $\left.\varepsilon^{*}\right)$ : SSB.

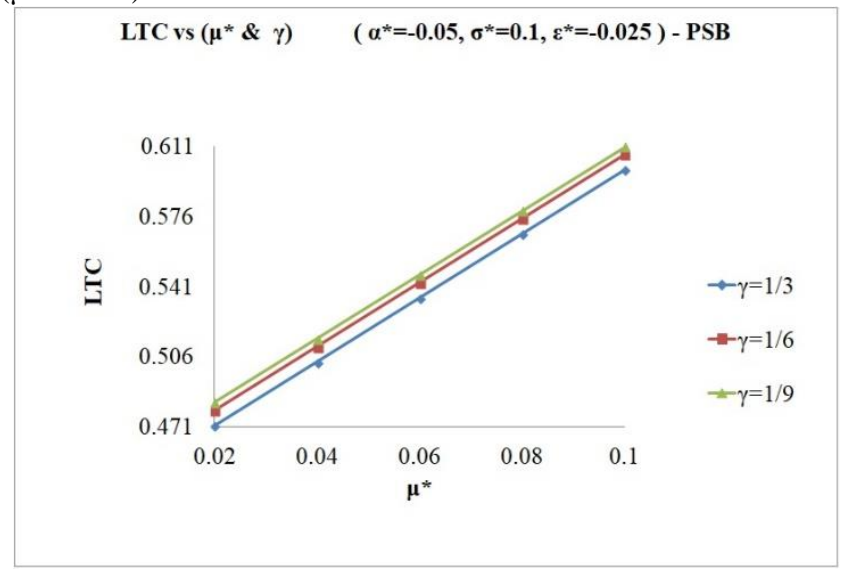

Fig. 16. LTC to ( $\mu^{*}$ and $\left.\gamma\right)$ : PSB.

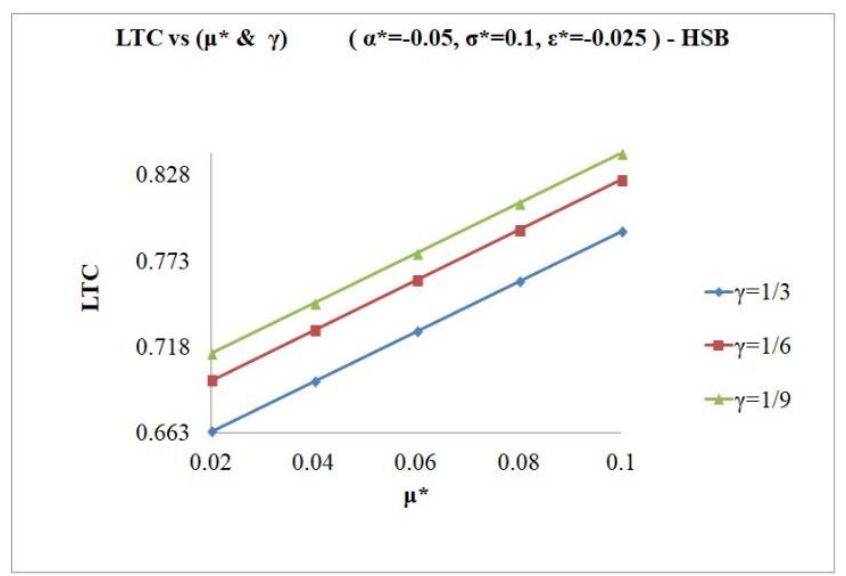

Fig. 17. LTC to ( $\mu^{*}$ and $\left.\gamma\right)$ : HSB. 


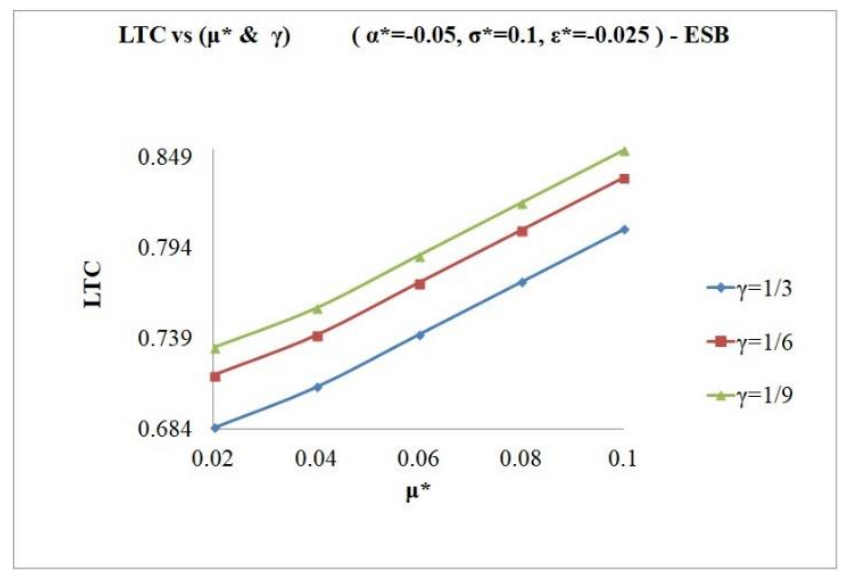

Fig. 18. LTC to $\left(\mu^{*}\right.$ and $\left.\gamma\right)$ : ESB.

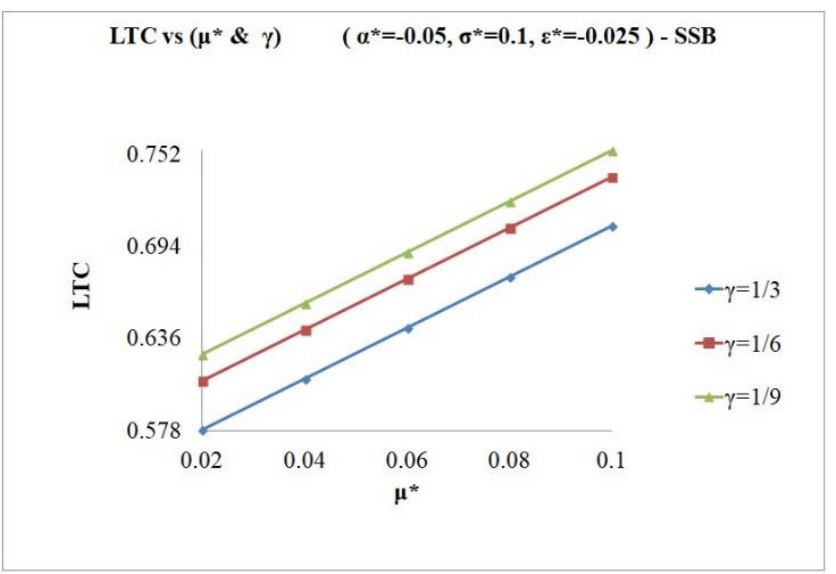

Fig. 19. LTC to ( $\mu^{*}$ and $\left.\gamma\right)$ SSB.

\section{Conclusion}

The current analysis asserts that the surface roughness, having negative skewness and negative mean produce better LTC regardless of the value of the $\operatorname{RPP}(\gamma)$. The ESB gives better performance than a PSB, HSB, and SSB. It is also to be noted that the LTC can be more boosted by applying the magnetic field at an appropriate area of the bearing system while using the Ferro-lubricant. This study shows that a less longitudinal rough slider bearing with $\alpha$ (-ve) and $\varepsilon$ (-ve) can be designed to overcome high friction and wear and achieve superior LTC. 


\section{Appendix A. nomenclature}

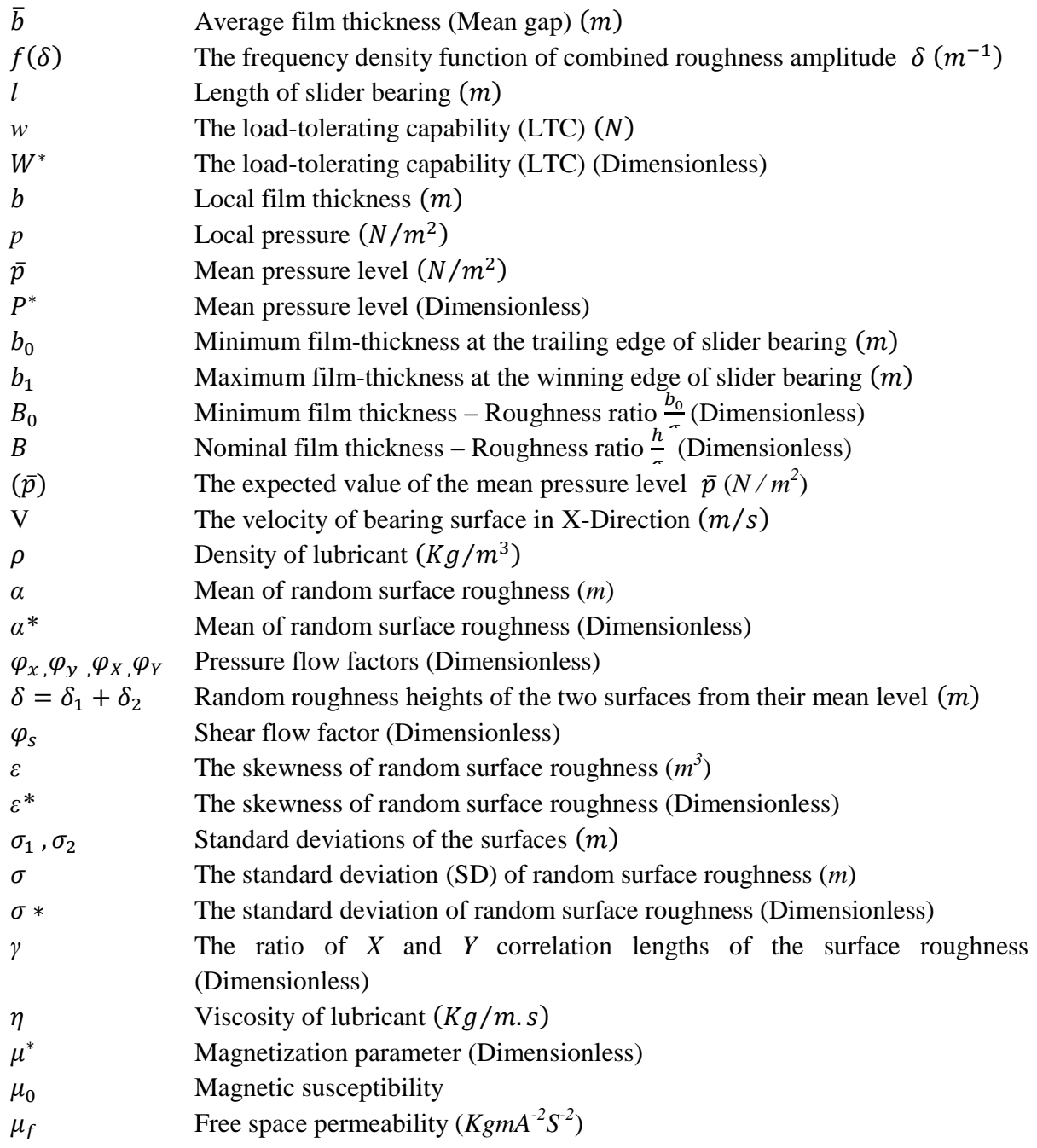

\section{References}

1. R. A. Burton, J. Fluids Eng. 85, 258 (1963). https://doi.org/10.1115/1.3656572

2. M. G. Davies, Lubr. Eng. 19, 246 (1963).

3. S. T. Tzeng and E. Saibel, ASLE Transactions 10, 334 (1967). https://doi.org/10.1080/05698196708972191

4. G. C. Panchal, G. M. Deheri, and H. C. Patel, Glob. J. Pure Appl. Math. 12, 783 (2016). https://doi.org/10.1155/2016/8197160

5. G. C. Panchal, G. M. Deheri, and H. C. Patel, Annals of the Faculty of Engineering HunedoaraInt. J. Eng. 14, 227 (2016).

6. G. C. Panchal, G. M. Deheri, and H. C. Patel, Int. J. Math. Sci. 34(A), 40 (2018). 
7. G. C. Panchal, G. M. Deheri, and H. C. Patel, The Effect of Magnetic Fluid Together with Transverse Roughness Pattern Parameters on the Performance of a Plane Slider Bearing, Numerical Modelling in Engineering, (Springer, Singapore, 2018) vol. 2, pp. 151. https://doi.org/10.1007/978-981-13-2273-0 13

8. N. S. Patel, D. P. Vakharia, G. M. Deheri, and H. C. Patel, Wear 376-377, 1877 (2017). https://doi.org/10.1016/j.wear.2017.01.113

9. H. A. Patel, M. P. Patel, H. C. Patel, and G. M. Deheri, Squeeze-Film Performance in Parallel Rough Circular Disks Lubricated by Ferro-fluid with Non-newtonian Couple Stress Effect Proc. of ICATES (2013) pp.111. https://doi.org/10.1007/978-81-322-1656-8_9

10. H. Christensen, Proc. Inst. Mech. Engineers 184, 1013 (1969). https://doi.org/10.1243/PIME_PROC_1969_184_074_02

11. H. Christensen and K. Tonder, J. Lubr. Tech. 93, 324 (1971). https://doi.org/10.1115/1.3451579

12. H. Christensen and K. Tonder, Proc. Inst. Mech. Engineers 186, 807 (1972). https://doi.org/10.1243/PIME_PROC_1972_186_096_02

13. H. A Christensen, Proc. Inst. Mech. Engineers 186, 421 (1972). https://doi.org/10.1243/PIME_PROC_1972_186_044_02

14. N. Patir, Ph.D. Thesis, Northwestern University, Illinois, USA (1978).

15. G. M. Deheri, P. I. Andharia, and R. M. Patel, Indust. Lubr. Tribol. 56, 177 (2004). https://doi.org/10.1108/00368790410532219

16. V. K. Agrawal, Wear 107, 133 (1986). https://doi.org/10.1016/0043-1648(86)90023-2 